\title{
Zerofinding of analytic functions by structured matrix methods
}

\author{
Luca Gemignani
}

\begin{abstract}
We propose a fast and numerically robust algorithm based on structured numerical linear algebra technology for the computation of the zeros of an analytic function inside the unit circle in the complex plane. At the core of our method there are two matrix algorithms: (a) a fast reduction of a certain linearization of the zerofinding problem to a matrix eigenvalue computation involving a perturbed CMV-like matrix and (b) a fast variant of the QR eigenvalue algorithm suited to exploit the structural properties of this latter matrix. We illustrate the reliability of the proposed method by several numerical examples
\end{abstract}

\section{Introduction}

Processes as population dynamics that evolve in discrete time steps are usually modeled using discrete dynamical systems. In this case the stability analysis for an equilibrium state of the system leads to the problem of computing all the zeros of a certain analytic or meromorphic function $f: \Omega \subseteq \mathbb{C} \rightarrow \mathbb{C}$ within a bounded domain in the complex plane. Ideally, increasingly accurate approximations of the zeros can be found after linearization by means of some efficient eigenvalue solver.

This approach is pursued in [3] where two linearization techniques based on the evaluation of $f(z)$ at roots of unity are investigated. Given the values $f_{k}=$ $f\left(e^{2 \pi(k-1) / n}\right), 1 \leq k \leq n$, attained by $f(z)$ at the $n$-th roots of unity, polynomial and rational interpolants of $f(z)$ are constructed whose zeros provide approximations of the zeros of $f(z)$ inside the unit circle. The resulting rootfinding computations can then be recast in a matrix setting as a (generalized) eigenproblem for companion-type matrices. If the coefficients of the interpolating polynomial are available then a customary companion matrix or pencil can be formed. Otherwise, working directly from values a generalized eigenvalue problem with arrowhead structure is defined [18, 28.

Although these two matrix eigenproblems are equivalent in the sense that they share the same finite spectrum, for the purpose of numerical computation we favor the second option based on the following reasons. First of all, the conditioning of the eigenvalues depends on the representation of the interpolating polynomial. From our numerical experience, the representation by values generally leads to a better conditioning for the approximations of the roots around the origin in the complex

2010 Mathematics Subject Classification. Primary 65H05, 30C15. 
plane. This part of the spectrum is relevant to establish the stability features of the system. A theoretical analysis supporting this claim is presented in [11. Secondly, for large $n$ the balancing of the vector of sampled values can directly be related with the behavior of the absolute value of the function on the unit circle. A similar conclusion does not hold for the coefficient vector which can be highly unbalanced even for small varying functions. Balancing is usually recommended for achieving efficiency and accuracy in numerical computations.

Whatever linearization technique we apply, it results in a rank-structured (generalized) eigenproblem. The exploitation of the structural properties of the resulting matrix eigenvalue problem also leads to a significant improvement of the computational efficiency. In recent years based on the concept of rank structure many authors have provided fast adaptations of the QR/QZ iteration applied to small rank modifications of Hermitian or unitary matrices/pencils [8, 9, 10, 17, 32, 20, 33, 12. However, despite the common framework, there are several differences between the Hermitian and the unitary case which makes the latter much more involved computationally. To circumvent this drawback in [7] a novel approach for solving the perturbed unitary eigenproblem has been proposed. The approach relies upon an initial transformation of the matrix by unitary congruence into a modified CMV-like form 15, 25 with staircase shape. The CMV-like form of a unitary matrix is particularly suited for the application of the QR eigenvalue algorithm 14. The staircase shape also reveals invariance properties under the same algorithm [2. Combining these facts together yields a very nice data-sparse parametrization of the matrix which is maintained under the iterative process.

In this paper we extend the CMV-based stuff to solving the generalized eigenproblem associated with the point-value representation of the interpolating polynomial of a given analytic function at the roots of unity. More specifically, we design a fast composite algorithm relying on the following two steps.

(1) The initial arrowhead pencil is converted into a modified CMV-like form. We propose a novel method for the reduction of a unitary diagonal matrix into a CMV-like form by unitary congruence having prescribed the first column of the associated transformation matrix. It turns out that this method applied to the initial matrix pair $(A, B)$ performs the reduction into a modified pair $(\widehat{A}, B)$, where $\widehat{A}$ is a CMV-like matrix perturbed by a rank-one correction in its first row. Then we adjust the deflation procedure described in $\mathbf{2 7}$ to the unitary setting by transforming the generalized eigenproblem $\widehat{A} \boldsymbol{x}=\lambda B \boldsymbol{x}$ into a classical eigenproblem $\tilde{A} \boldsymbol{x}=$ $\lambda \boldsymbol{x}$ having the same finite spectrum. In addition, the matrix $\tilde{A}$ still inherits the modified CMV-like structure of $\widehat{A}$. The complexity of the reduction process is quadratic in the size of the matrix.

(2) The fast adaptation of the QR scheme proposed in [7] is applied for the solution of the resulting matrix eigenproblem involving $\tilde{A}$. The modified CMV-like structure of $\tilde{A}$ induces the rank structure of the matrices generated under the QR process applied to $\tilde{A}$. By using a suitable entrywise parametrization of these matrices each iteration can be carried out at a linear time so that the overall complexity remains quadratic.

The composite algorithm is numerically reliable, since each computational step relies on unitary transformations. The computational efficiency is enhanced by the use of data-sparse representations of the involved matrices that are easy to 
manipulate and update. The overall complexity is quadratic using a linear memory storage.

A detailed outline of the paper is as follows. In Section 2 we formulate the computational problem and develop our algorithm for performing the reductions carried out at step 1. In Section 3 we briefly restate the fast QR iteration presented in 7. In Section 4 we discuss several numerical examples indicating the robustness and the efficiency of the proposed numerical approach. Finally, the conclusion and further developments are drawn in Section 5 .

\section{Problem Statement and Basic Reductions}

In this section we first introduce the matrix eigenproblem with arrowhead shape resulting from the polynomial interpolation at the roots of unity of an analytic function $f(z)$. Then, we describe the basic reductions of this problem into a modified CMV-like form.

The unique polynomial of degree less than $n$ interpolating the function $f(z)$ at the $n$-th roots of unity $z_{k}=e^{2 \pi(k-1) / n}, 1 \leq k \leq n$, can be expressed as

$$
p(z)=\left(z^{n}-1\right) \sum_{j=1}^{n} \frac{w_{j} f_{j}}{z-z_{j}},
$$

where

$$
f_{j}=f\left(z_{j}\right), \quad w_{j}=\left(\prod_{k=1, k \neq j}\left(z_{j}-z_{k}\right)\right)^{-1}=z_{j} / n, \quad 1 \leq j \leq n .
$$

In $[18$ it was shown that the roots of $p(z)$ are the finite eigenvalues of the matrix pencil given by

$$
T(z)=A-z B, \quad A, B \in \mathbb{C}^{(n+1) \times(n+1)},
$$

where

$$
A=\left[\begin{array}{cccc}
0 & -\xi_{1} f_{1} & \ldots & -\xi_{n} f_{n} \\
w_{1} / \xi_{1} & z_{1} & & \\
\vdots & & \ddots & \\
w_{n} / \xi_{n} & & & z_{n}
\end{array}\right], \quad B=\left[\begin{array}{cccc}
0 & & & \\
& 1 & & \\
& & \ddots & \\
& & & 1
\end{array}\right]
$$

where $\xi_{1}, \ldots, \xi_{m}$ are nonzero additional parameters introduced for balancing and symmetry purposes. Observe that since the size of the matrices $A, B$ is $n+1$, then $T(z)$ in 2.1), 2.2 has at least two spurious infinite eigenvalues. In the sequel of this section we present a method to transform the generalized eigenproblem for the matrix pair (2.2) into a classical eigenvalue problem for a perturbed CMV-like matrix. This reduction also incorporates a reliable numerical technique for deflating some possibly infinite eigenvalues.

CMV matrices, introduced in [15] and [30] where the term was coined, are defined as product of Givens transformations arranged in suitable patterns. For a given pair $(\gamma, k) \in \mathbb{D} \times \mathbb{I}_{n}, \mathbb{D}=\{z \in \mathbb{C}:|z|<1\}, \mathbb{I}_{n}=\{1,2, \ldots, n-1\}$, we set

$$
\mathcal{G}_{k}(\gamma)=I_{k-1} \oplus\left[\begin{array}{cc}
\bar{\gamma} & \sigma \\
\sigma & -\gamma
\end{array}\right] \oplus I_{n-k-1} \in \mathbb{C}^{n \times n}
$$


where $\sigma \in \mathbb{R}, \sigma>0$ and $|\gamma|^{2}+\sigma^{2}=1$. Similarly, if $\gamma \in \mathbb{S}^{1}=\{z \in \mathbb{C}:|z|=1\}$ then denote

$$
\mathcal{G}_{n}(\gamma)=I_{n-1} \oplus \gamma \in \mathbb{C}^{n \times n} .
$$

The following definition identifies an important class of structured unitary matrices.

Definition 2.1. 15] For a given coefficient sequence $\left(\gamma_{1}, \ldots, \gamma_{n-1}, \gamma_{n}\right) \in$ $\mathbb{D}^{n-1} \times \mathbb{S}^{1}$ we introduce the unitary block diagonal matrices

$\mathcal{L}=\mathcal{G}_{1}\left(\gamma_{1}\right) \cdot \mathcal{G}_{3}\left(\gamma_{3}\right) \cdots \mathcal{G}_{2\left\lfloor\frac{n+1}{2}\right\rfloor-1}\left(\gamma_{2\left\lfloor\frac{n+1}{2}\right\rfloor-1}\right), \quad \mathcal{M}=\mathcal{G}_{2}\left(\gamma_{2}\right) \cdot \mathcal{G}_{4}\left(\gamma_{4}\right) \cdots \mathcal{G}_{2\left\lfloor\frac{n}{2}\right\rfloor}\left(\gamma_{2\left\lfloor\frac{n}{2}\right\rfloor}\right)$, and define

$$
\mathcal{C}=\mathcal{L} \cdot \mathcal{M}
$$

as the CMV matrix associated with the prescribed coefficient list.

The decomposition (2.4) of a unitary matrix was first investigated for eigenvalue computation in $\mathbf{1}$ and 14]. The shape of CMV matrices is analyzed in [25] where the next definition is given.

Definition 2.2. 25] A matrix $F \in \mathbb{C}^{n \times n}$ has CMV shape if the possibly nonzero entries exhibit the following pattern where + denotes a positive entry:

$$
F=\left[\begin{array}{ccccccccc}
\star & \star & + & & & & & \\
+ & \star & \star & & & & & \\
& \star & \star & \star & + & & & \\
& + & \star & \star & \star & & & \\
& & & \star & \star & \star & + & \\
& & & + & \star & \star & \star & \\
& & & & & \star & \star & \star \\
& & & & & + & \star & \star
\end{array}\right], \quad(n=2 k),
$$

or

$$
F=\left[\begin{array}{ccccccc}
\star & \star & + & & & & \\
+ & \star & \star & & & & \\
& \star & \star & \star & + & & \\
& + & \star & \star & \star & & \\
& & & \star & \star & \star & + \\
& & & + & \star & \star & \star \\
& & & & & \star & \star
\end{array}\right], \quad(n=2 k-1) .
$$

The definition is useful for computational purposes since shapes are easier to check than comparing the entries of the matrix. Obviously, CMV matrices have a CMV shape and, conversely, it is shown that a unitary matrix with CMV shape is CMV [16. The positiveness of the complementary parameters $\sigma_{k}$ in $(2.3)$ as well as of the entries marked with + in Definition 2.2 is necessary to establish the connection of CMV matrices with corresponding sequences of orthogonal polynomials on the unit circle [25. From the point of view of eigenvalue computation, however, this condition can be relaxed. In [6] we simplify the above definition by skipping the positiveness condition on the entries denoted as + . The fairly more general class of matrices considered in [6] is referred to as CMV-like matrices. There it is also shown that the block Lanczos method can be used to reduce a unitary matrix into the direct sum of CMV-like matrices. Here we pursue a different approach relying upon the fact that the reduction of the arrow matrix pencil $2.1, \sqrt{2.2}$ into 
a perturbed CMV-like form follows from computing a unitary matrix $Q \in \mathbb{C}^{n \times n}$ such that

$$
Q^{H} D Q=F, \quad Q^{H} \boldsymbol{w}=\alpha \boldsymbol{e}_{1},
$$

where $D=\operatorname{diag}\left[z_{1}, \ldots, z_{n}\right], F$ is CMV-like and, moreover,

$$
A=\left[\begin{array}{cccc}
0 & -\xi_{1} f_{1} & \ldots & -\xi_{n} f_{n} \\
w_{1} / \xi_{1} & z_{1} & & \\
\vdots & & \ddots & \\
w_{n} / \xi_{n} & & & z_{n}
\end{array}\right]=\left[\begin{array}{c|c}
0 & -\boldsymbol{f}^{H} \\
\hline \boldsymbol{w} & D
\end{array}\right] .
$$

Observe that the bordered unitary matrix

$$
\widehat{Q}=\left[\begin{array}{l|l}
1 & \mathbf{0}^{T} \\
\hline \mathbf{0} & Q
\end{array}\right]
$$

satisfies

$$
\widehat{Q}^{H} A \widehat{Q}=\left[\begin{array}{c|c}
0 & \widehat{\boldsymbol{f}}^{H} \\
\hline \alpha \boldsymbol{e}_{1} & F
\end{array}\right], \quad \widehat{Q}^{H} B \widehat{Q}=B .
$$

In addition from (2.5) there follows that

$$
Q^{H} D \boldsymbol{w}=F Q^{H} \boldsymbol{w}=\alpha F \boldsymbol{e}_{1}
$$

which means that the entries of $Q^{H} D \boldsymbol{w}$ from 3 to $n$ are zero.

In the next subsection we present an efficient solution of 2.5 based on the recognition of 2.5 as an inverse eigenvalue problem (IEP) associated with the reduction to $\mathrm{CMV}$-like form.

2.1. Efficient Recursive Solution of the Associated IEP. The solvability of (2.5) is addressed in [6 by using a constructive approach relying upon the blockLanczos method. We just recall the main result here. For the sake of simplicity we consider the case where $n$ is even.

THEOREM 2.3. Let $\mathcal{D}_{+}=\frac{D+D^{H}}{2}$ and $\mathcal{D}_{-}=\frac{D-D^{H}}{2}$ be such that $D=\mathcal{D}_{+}+$ $\mathcal{D}_{-}$. Then the block-Lanczos procedure applied to $\mathcal{D}_{+}$with initial vectors $[\boldsymbol{w} \mid D \boldsymbol{w}]$ produces a unitary matrix $Q \in \mathbb{C}^{n \times n}$ satisfying:

(1) $[\boldsymbol{w} \mid D \boldsymbol{w}]=Q(:, 1: 2) \cdot R$ for an upper triangular $R$;

(2) $Q^{H} \mathcal{D}_{+} Q, Q^{H} \mathcal{D}_{-} Q$ and, a fortiori, $Q^{H} D Q$ are block tridiagonal matrices with $2 \times 2$ diagonal and off-diagonal blocks.

(3) By denoting with $B_{i}, C_{i}, 1 \leq i \leq n / 2-1$, the subdiagonal and superdiagonal blocks, respectively, in the block tridiagonal reduction of $D$ it is found that rank $\left(B_{i}\right) \leq 1$ and $\operatorname{rank}\left(C_{i}\right) \leq 1$ and, moreover,

$$
B_{i}=\left[\begin{array}{cc}
0 & \star \\
0 & \star
\end{array}\right], \quad C_{i}=\left[\begin{array}{cc}
\star & 0 \\
\star & 0
\end{array}\right],
$$

where $\star$ denotes a possibly nonzero entry.

The construction advocated in the previous theorem can suffer from numerical instabilities and premature breakdowns demanding for restarting techniques. An Householder-style implementation of the Lanczos reduction is described in [25]. An alternative approach based on the Householder (block) tridiagonalization algorithm applied to $\mathcal{D}_{+}$is also presented in [6] in order to circumvent these difficulties. Hereafter we further elaborate on this modification by devising a novel reduction 
scheme using unitary transformations which has the advantage of working directly on the matrix $D$ rather than $\mathcal{D}_{+}$. Our approach relies upon the recognition of 2.5 as an inverse eigenvalue problem (IEP). Recursive solution methods are customary in this field (see [13, 31 and the references given therein) and can be extended to deal with the present case.

The construction of $Q$ can be carried out by a recursive algorithm which acts on the enlarged matrix

$$
\mathbb{C}^{(n+2) \times(n+2)} \ni \widehat{A}=\left[\begin{array}{cc|c}
0 & 0 & \mathbf{0}^{T} \\
0 & 0 & -\boldsymbol{f}^{H} \\
\hline \boldsymbol{w} & D \boldsymbol{w} & D
\end{array}\right]
$$

by annihilating entries of the first two columns and then by returning the trailing submatrix to CMV-like form. For the sake of simplicity we restrict ourselves to the case where $n$ is even. The procedure is recursive. Suppose that $Q_{n-k} \in \mathbb{C}^{(n-k) \times(n-k)}$ solves 2.5 for the input data $D_{n-k}=D(k+1: n, k+1: n)$ and $\boldsymbol{w}^{(n-k)}=\boldsymbol{w}(k+1: n)$, that is,

$$
Q_{n-k}^{H} D_{n-k} Q_{n-k}=F_{n-k}, \quad Q_{n-k}^{H} \boldsymbol{w}^{(n-k)}=\alpha_{n-k} \boldsymbol{e}_{1} .
$$

It is found that the unitary matrix $\widehat{Q}_{n-k}=I_{k+2} \oplus Q_{n-k}$ satisfies $\widehat{Q}_{n-k}^{H} \widehat{A} \widehat{Q}_{n-k}=$ $\widehat{A}_{n-k}$, where

$$
\widehat{A}_{n-k}(k-1: n, 1: n+2)=\left[\begin{array}{l|ll|l}
W_{n-k} & z_{k-1} & \\
\hline R_{n-k} & & z_{k} & \\
& & F_{n-k}
\end{array}\right],
$$

where $W_{n-k}, R_{n-k} \in \mathbb{C}^{2 \times 2}$ and $R_{n-k}$ is upper triangular. Then we can determine a unitary matrix $\mathcal{G}_{n-k}$ such that

$$
\mathcal{G}_{n-k}^{H}\left[\begin{array}{c}
W_{n-k} \\
R_{n-k}
\end{array}\right]=\widehat{R}_{n-k-2}=\left[\begin{array}{cc}
R_{n-k+2} \\
0 & 0 \\
0 & 0
\end{array}\right]
$$

Let

$$
\left(I_{k} \oplus \mathcal{G}_{n-k} \oplus I_{n-k-2}\right)^{H} \widehat{A}_{n-k}\left(I_{k} \oplus \mathcal{G}_{n-k} \oplus I_{n-k-2}\right)=\tilde{A}_{n-k}
$$

be partitioned as follows

$$
\tilde{A}_{n-k}(k-1: n, 1: n+2)=\left[\begin{array}{l|l|l}
R_{n-k+2} & O_{n-k+2, k-2} & \tilde{F}_{n-k+2}
\end{array}\right],
$$

where

$$
\tilde{F}_{n-k+2}=\left(\mathcal{G}_{n-k} \oplus I_{n-k-2}\right)^{H}\left[\begin{array}{ll|l}
z_{k-1} & & \\
& z_{k} & \\
\hline & & F_{n-k}
\end{array}\right]\left(\mathcal{G}_{n-k} \oplus I_{n-k-2}\right) .
$$

By denoting

this implies that

$$
\hat{Q}_{n-k+2}=\left(I_{2} \oplus Q_{n-k}\right) \cdot\left(\mathcal{G}_{n-k} \oplus I_{n-k-2}\right),
$$

$$
\hat{Q}_{n-k+2}^{H} D_{n-k+2} Q_{n-k+2}=\tilde{F}_{n-k+2},
$$

and, moreover,

$$
\hat{Q}_{n-k+2}^{H} \boldsymbol{w}^{(n-k+2)}=\left(R_{n-k+2}\right)_{1,1} \boldsymbol{e}_{1},
$$




$$
\hat{Q}_{n-k+2}^{H} D_{n-k+2} \boldsymbol{w}^{(n-k+2)}=\left(R_{n-k+2}\right)_{1,2} \boldsymbol{e}_{1}+\left(R_{n-k+2}\right)_{2,2} \boldsymbol{e}_{2} .
$$

Now, the following properties can easily be deduced.

(1) As $\tilde{F}_{n-k+2}, \hat{Q}_{n-k+2}$ fulfill (2.6), 2.7) and 2.8) for a partial set of input data we obtain that the first column of $\tilde{F}_{n-k+2}$ is of the form $\rho \boldsymbol{e}_{1}+\xi \boldsymbol{e}_{2}$ for suitable $\rho, \xi$.

(2) Since $\tilde{F}_{n-k+2}$ is unitary, from the nullity theorem 22 this also implies that $\tilde{F}_{n-k+2}(1: 2,3: n-k+2)$ is a matrix of rank one at most.

(3) As $\tilde{F}_{n-k+2}(1: 2,4: 5)$ is annihilated by a unitary transformation on the right then due to the nullity theorem a staircase pattern is identified in the lower triangular portion of the modified $\tilde{F}_{n-k+2}$ and, therefore, the rank properties are transmitted to the trailing submatrices.

By combining these three facts together we find that there exists a matrix $\tilde{Q}_{n-k+2}$ such that $\tilde{Q}_{n-k+2}^{H} \tilde{F}_{n-k+2} \tilde{Q}_{n-k+2}=F_{n-k+2}$ is a CMV-like matrix and, moreover, $\tilde{Q}_{n-k+2}$ only acts on the columns of $\tilde{F}_{n-k+2}$ in position 3 through $n-k+2$. And thus, finally, by setting

$$
Q_{n-k+2}=\hat{Q}_{n-k+2} \cdot \tilde{Q}_{n-k+2},
$$

we may conclude that this latter matrix solves 2.5 for the extended set of input data $D_{n-k+2}$ and $\boldsymbol{w}^{(n-k+2)}$.

The complete reduction scheme is stated below in algorithmic form. For the sake of notational convenience we assume to do nothing whenever index exceeds matrix dimensions.

$$
\begin{aligned}
& \text { Procedure CMV_Reduce } \\
& \text { Input: } n=2 k, n 2=n+2, \boldsymbol{w}, \boldsymbol{f} \in \mathbb{C}^{n} \text {; } \\
& \text { Output: } Q, F \in \mathbb{C}^{n \times n} \text { as in } 2.5 \text {; } \\
& \text { for } k=n 2:-2: 6 \\
& E=\widehat{A}(k-3: k, 1: 2) ; \quad[Q, R]=\operatorname{qr}(E) ; \\
& \widehat{A}(k-3: k,:)=Q^{H} \widehat{A}(k-3: k,:) ; \widehat{A}(:, k-3: k)=\widehat{A}(:, k-3: k) Q ; \\
& \text { for } j=k: 2: n 2 \\
& E=\widehat{A}(j-3: j-2, j: j+1) ; E=E^{H} ; \quad[Q, R]=\operatorname{qr}(E) ; \\
& \widehat{A}(:, j: j+1)=\widehat{A}(:, j: j+1) Q ; \widehat{A}(j: j+1,:)=Q^{H} \widehat{A}(j: j+1,:) ; \\
& E=\widehat{A}(j+1: j+2, j-2) ; \quad[Q, R]=\operatorname{qr}(E) ; \tilde{j}=j+1 \\
& \widehat{A}(:, \tilde{j}: \tilde{j}+1)=\widehat{A}(:, \tilde{j}: \tilde{j}+1) Q ; \widehat{A}(\tilde{j}: \tilde{j}+1,:)=Q^{H} \widehat{A}(\tilde{j}: \tilde{j}+1,:) ; \\
& E=\widehat{A}(j-3: j-2, j-1: j) ; E=E^{H} ; \quad[Q, R]=\operatorname{qr}(E) ; \\
& \widehat{A}(:, j-1: j)=\widehat{A}(:, j-1: j) Q ; \widehat{A}(j-1: j,:)=Q^{H} \widehat{A}(j-1: j,:) ; \\
& E=\widehat{A}(j: j+1, j-2) ; \quad[Q, R]=\operatorname{qr}(E) ; \\
& \widehat{A}(:, j: j+1)=\widehat{A}(:, j: j+1) Q ; \widehat{A}(j: j+1,:)=Q^{H} \widehat{A}(j: j+1,:) ; \\
& \text { end } \\
& \text { end }
\end{aligned}
$$

Since each computation involves unitary matrices of size $m \leq 4$ and the sparse form of $\widehat{A}$ is restored at each step it is easy to show that $\mathbf{C M V} \mathbf{V}$ Reduce has a cost of $O\left(n^{2}\right)$ flops. For the sake of illustration in Figure 1 we visualize the sparsity pattern of the matrix $\widehat{A}$ generated by the $\mathbf{C M V}$ _Reduce procedure at different times. 


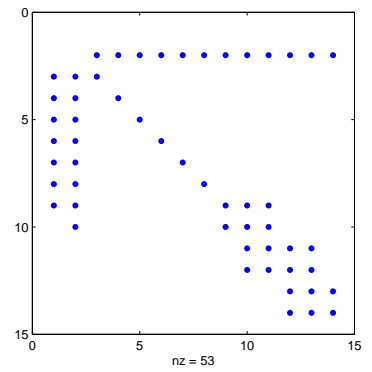

(a) $k=12$

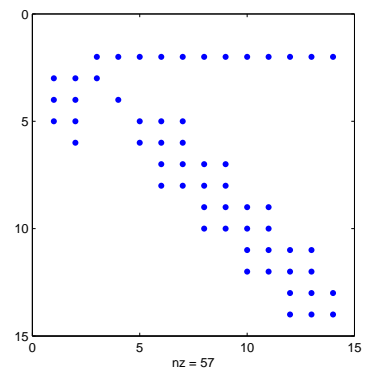

(c) $k=8$

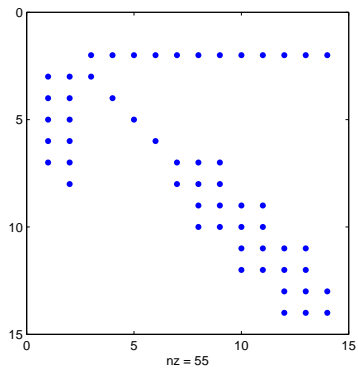

(b) $k=10$

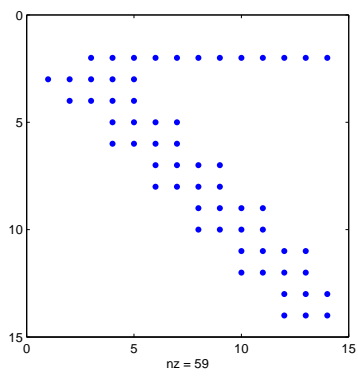

(d) $k=6$

Figure 1. Sparsity pattern of $\widehat{A}$ of size $n 2=14$ at the end of the for cycle with $k=n 2-2, n 2-4, n 2-6$ and $n 2-8$.

We conclude this section with two remarks concerning the reduction of 2.2 into a CMV-like pencil.

REMARK 2.4. It should be worth noticing that if $\xi_{1}=z_{1}, \ldots, \xi_{n}=z_{n}$ and $\boldsymbol{w}=(1 / n)[1, \ldots, 1]^{T}$ then an alternative reduction scheme of 2.2$)$ into a CMV-like pencil follows from the properties of the Fourier matrix $\Omega=(1 / \sqrt{n})\left(\omega^{(i-1)(j-1)}\right)$ 
with $\omega=z_{2}$. In fact it is well known that

$$
\Omega^{H} D \Omega=\left[\begin{array}{cccc}
0 & & & 1 \\
1 & 0 & & 0 \\
& \ddots & \ddots & \vdots \\
& & 1 & 0
\end{array}\right], \quad \Omega^{H} \boldsymbol{w}=\alpha e_{1} .
$$

Then by making use of the permutation matrix $P$ defined in [7] it can easily be seen that $Q=\Omega P$ solves 2.5 and, hence, $Q=1 \oplus \Omega P$ converts the input arrowhead pencil 2.1], 2.2), into the permuted companion form considered in [7].

REMark 2.5. The Lanczos methods proposed in [6] and 25] for the reduction of a unitary matrix to CMV-like form can break down for suitable choices of the vector $\boldsymbol{w}$ or when the starting matrix has multiple eigenvalues. It is clear that the possibly coalescence of nodes produces degeneracies also in previous algorithm CMV_Reduce.

2.2. Deflation Technique. Once the modified matrix pair $\left(A_{1}, B_{1}\right), A_{1}=$ $\widehat{Q}^{H} A \widehat{Q}, B_{1}=B$, has been constructed then a procedure like that one proposed in [27] can be carried out in order to deflate the two spurious infinite eigenvalues. Specifically we proceed in the following steps.

(1) At first, we swap the first two rows of $A_{1}$ and $B_{1}$ by using a permutation matrix $P_{1}$ on the left. Since $\widehat{A}_{1}=P_{1} A_{1}$ is block upper triangular and $\widehat{B}_{1}=P_{1} B_{1}$ is upper triangular we can delete the first row and column of $\widehat{A}_{1}$ and $\widehat{B}_{1}$ by obtaining the novel matrix pair $\left(A_{2}, B_{2}\right)$ with $A_{2}=$ $\widehat{A}_{1}(2: n+1 ; 2: n+1)$ and $B_{2}=\widehat{B}_{1}(2: n+1 ; 2: n+1)$. Observe that $A_{2}$ can be expressed as a CMV-like unitary matrix perturbed by a rank-one correction located in its first row.

(2) Then we determine a $2 \times 2$ Givens rotation matrix which acts on the first two rows of $A_{2}$ by annihilating the first subdiagonal entry. By applying the same transformation to $B_{2}$ we find that the modified matrices $\widehat{A}_{2}$ and $\widehat{B}_{2}$ are in block upper triangular and upper triangular form, respectively. Thus we can again perform a deflation step by removing the second spurious infinite eigenvalue and reducing the size of $A_{2}$ and $B_{2}$ by one. The final matrix pair is $\left(A_{3}, B_{3}\right)$, where $A_{3}, B_{3} \in \mathbb{C}^{(n-1) \times(n-1)}$ and

$$
B_{3}=\left[\begin{array}{llll}
\sigma & & & \\
& 1 & & \\
& & \ddots & \\
& & & 1
\end{array}\right],
$$

for a possibly nonzero scalar $\sigma$. Also it should be noticed that $A_{3}$ can be further written as a rank-one modification of a CMV-like unitary matrix so that at the very end of this process whenever $\sigma \neq 0$ we obtain that the spectrum of the structured matrix $B_{3}^{-1} A_{3}$ gives suitable approximations of the zeros of $p(z)$.

Summing up, if the deflation procedure succeeds then the generalized eigenproblem $A_{1} \boldsymbol{x}=\lambda B_{1} \boldsymbol{x}$ is reduced to the classical eigenvalue problem $A \boldsymbol{x}=\lambda \boldsymbol{x}$, where $A$ : $=B_{3}^{-1} A_{3}$ is a CMV-like unitary matrix perturbed by a rank-one correction located in its first row. The structure of this latter matrix will be exploited 
in the next section for the design of an efficient eigensolver. It is worth pointing out that theoretically, if $\sigma=0$ then the deflation procedure may be continued by preserving the structural properties of the pencil. However, in practice, from a numerical point of view the critical issue to be addressed is the occurrence of a quite small but nonzero value of $\sigma$. We will go back on this later in Section 4 .

\section{A Fast Structured QR Algorithm}

In this section we are going to develop a fast variant of the customary QR eigenvalue algorithm for an input matrix $A=F-\boldsymbol{e}_{1} \boldsymbol{w}^{H}$ which can be represented as a CMV-like unitary matrix plus a rank-one correction located in the first row. The derivation follows largely that in [7, where the interested reader is directed for more details. Our approach is able to exploit both the staircase form of $A$ and its perturbed $\mathrm{CMV}$-like representation.

Staircase matrix patterns can be exploited for eigenvalue computation [2. The shifted QR algorithm

$$
\left\{\begin{array}{l}
A_{s}-\rho_{s} I_{n}=Q_{s} R_{s} \\
A_{s+1}=Q_{s}^{H} A_{s} Q_{s}, \quad s \geq 0
\end{array}\right.
$$

is the standard algorithm for computing the Schur form of a general matrix $A=$ $A_{0} \in \mathbb{C}^{n \times n}$ 23]. The matrix $A$ is said to be staircase if $m_{j}(A) \geq m_{j-1}(A)$, $2 \leq j \leq n$, where

$$
m_{j}(A)=\max \left\{j, \max _{i>j}\left\{i: a_{i, j} \neq 0\right\}\right\} .
$$

The staircase form is preserved under the QR iteration (3.1) in the sense that [2]

$$
m_{j}\left(A_{s+1}\right) \leq m_{j}\left(A_{s}\right), \quad 1 \leq j \leq n .
$$

For Hermitian and unitary matrices the staircase form also implies a zero pattern or a rank structure in the upper triangular part. The invariance of this pattern by the QR algorithm is proved in [2] for Hermitian matrices and in [14] for unitary CMV-shaped matrices. An alternative proof for the unitary case that is suitable for generalizations is given in $\mathbf{6}$ by relying upon the classical nullity theorem $\mathbf{2 2}$.

It has already been noticed above that the staircase form of $A_{0}=: A$ is preserved under the shifted QR iteration (3.1). This means that each unitary matrix $Q_{s}$ is also in staircase form. From

$$
A_{0}=F-\boldsymbol{e}_{1} \boldsymbol{w}^{H}=F_{0}-\boldsymbol{z}_{0} \boldsymbol{w}_{0}^{H}
$$

it follows that

$$
A_{s+1}=Q_{s}^{H} A_{s} Q_{s}=Q_{s}^{H}\left(F_{s}-\boldsymbol{z}_{s} \boldsymbol{w}_{s}^{H}\right) Q_{s}=F_{s+1}-\boldsymbol{z}_{s+1} \boldsymbol{w}_{s+1}^{H}, \quad s \geq 0,
$$

where

$$
F_{s+1}:=Q_{s}^{H} F_{s} Q_{s}, \quad \boldsymbol{z}_{s+1}:=Q_{s}^{H} \boldsymbol{z}_{s}, \quad \boldsymbol{w}_{s+1}:=Q_{s}^{H} \boldsymbol{w}_{s} .
$$

These relations enable the entries of each $A_{s}$ to be represented in terms of a linear number of parameters. Hereafter we recall the main result in [7].

Theorem 3.1. For any $s \geq 0$ the unitary matrix $F_{s}$ satisfies

$$
\operatorname{rank}\left(F_{s}(1: 2 j, 2(j+1)+1: n)\right) \leq 1, \quad 1 \leq j \leq\left\lfloor\frac{n+1}{2}\right\rfloor-2, s \geq 0 .
$$

Moreover, if $A_{0}$ is invertible then

$F_{s}(1: 2 j, 2(j+1)+1: n)=B_{s}(1: 2 j, 2(j+1)+1: n), \quad 1 \leq j \leq\left\lfloor\frac{n+1}{2}\right\rfloor-2, s \geq 0$, 
where

$$
B_{s}=\frac{F_{s} \boldsymbol{w}_{s} \boldsymbol{z}_{s}^{H} F_{s}}{\boldsymbol{z}_{s}^{H} F_{s} \boldsymbol{w}_{s}-1}=Q_{s}^{H} B_{s-1} Q_{s}, \quad s \geq 1,
$$

is a rank one matrix.

From the previous theorem we derive a structural representation of each matrix $A_{s}, s \geq 0$, generated under the QR process (3.1) applied to $A_{0}$. Let us start by observing that each matrix $A_{s}, s \geq 0$, generated by (3.1) can be represented by means of the following sparse data set of size $O(n)$ :

(1) the nonzero entries of the banded matrix $\widehat{A}_{s} \in \mathbb{C}^{n \times n}$ obtained from $A_{s}$ according to

$$
\widehat{A}_{s}=\left(\hat{a}_{i, j}^{(s)}\right), \quad \hat{a}_{i, j}^{(s)}=\left\{\begin{array}{l}
0, \text { if } j \geq 2\left\lfloor\frac{i+1}{2}\right\rfloor+3,1 \leq i \leq 2\left\lfloor\frac{n+1}{2}\right\rfloor-4 \\
a_{i, j}^{(s)}, \text { elsewhere }
\end{array}\right.
$$

(2) the vectors $\boldsymbol{z}_{s}=\left(z_{i}^{(s)}\right), \boldsymbol{w}_{s}=\left(w_{i}^{(s)}\right) \in \mathbb{C}^{n}$ and $\boldsymbol{f}_{s}:=F_{s} \boldsymbol{w}_{s}, \boldsymbol{f}_{s}=\left(f_{i}^{(s)}\right)$, and $\boldsymbol{g}_{s}:=F_{s}^{H} \boldsymbol{z}_{s}, \boldsymbol{g}_{s}=\left(g_{i}^{(s)}\right)$.

The nonzero pattern of the matrix $\widehat{A}_{s}$ looks as below:

$$
\widehat{A}_{s}=\left[\begin{array}{ccccccccc}
\star & \star & \star & \star & & & & \\
\star & \star & \star & \star & & & & \\
& \star & \star & \star & \star & \star & & \\
& \star & \star & \star & \star & \star & & \\
& & & \star & \star & \star & \star & \star \\
& & & \star & \star & \star & \star & \star \\
& & & & & \star & \star & \star \\
& & & & & \star & \star & \star
\end{array}\right], \quad(n=2 k),
$$

or

$$
\widehat{A}_{s}=\left[\begin{array}{cccccccc}
\star & \star & \star & \star & & & \\
\star & \star & \star & \star & & & \\
& \star & \star & \star & \star & \star & \\
& \star & \star & \star & \star & \star & \\
& & & \star & \star & \star & \star \\
& & & \star & \star & \star & \star \\
& & & & & \star & \star
\end{array}\right], \quad(n=2 k-1) .
$$

From (3.2) and 3.4 we find that the entries of the matrix $A_{s}=\left(a_{i, j}^{(s)}\right)$ can be expressed in terms of elements of this data set as follows:

$$
a_{i, j}^{(s)}=\left\{\begin{array}{l}
-\sigma^{-1} f_{i}^{(s)} \bar{g}_{j}^{(s)}-z_{i}^{(s)} \bar{w}_{j}^{(s)}, \text { if } j \geq 2\left\lfloor\frac{i+1}{2}\right\rfloor+3,1 \leq i \leq 2\left\lfloor\frac{n+1}{2}\right\rfloor-4 \\
\widehat{a}_{i, j}^{(s)}, \text { elsewhere }
\end{array}\right.
$$

where $\sigma=1-\boldsymbol{z}_{s}^{H} F_{s} \boldsymbol{w}_{s}=1-\boldsymbol{z}_{0}^{H} F_{0} \boldsymbol{w}_{0}$.

A fast adaptation of the QR iteration (3.1) applied to a starting invertible matrix $A_{0}=F-\boldsymbol{e}_{1} \boldsymbol{w}^{H} \in \mathbb{C}^{n \times n}$ by using the structural properties described above is devised in [7. At each step this method works on a condensed entrywise datasparse representation of the matrix using $O(n)$ flops and $O(n)$ memory storage. An efficient MatLal[1 implementation of the fast iteration called Fast_QR is also

\footnotetext{
${ }^{1}$ Matlab is a registered trademark of The MathWorks, Inc..
} 
provided in [7. This implementation is one main building block of our composite algorithm for the approximation of the zeros of an analytic function presented in the next section.

\section{Numerical Results}

Based on the results stated in the previous sections we propose the following composite algorithm for the computation of the zeros of an analytic function $f: \mathbb{C} \rightarrow$ $\mathbb{C}$ inside the unit circle in the complex plane.

(1) For a given $n \in \mathbb{N}$ form the arrowhead pair $(A, B)$ defined in $(2.1), 2.2$, where for balancing issues the additional parameters $\xi_{j}$ are set to $\xi_{j}=$ $1 / \sqrt{\left|f\left(z_{j}\right)\right|}$ for $f\left(z_{j}\right) \neq 0$.

(2) Apply the procedure $\mathbf{C M V}$ _Reduce by transforming the input arrowhead pair $(A, B)$ into $\left(A_{1}, B\right)$ where $A_{1}$ is a CMV-like matrix modified by a rank-one correction in its first row.

(3) Apply the deflation procedure in order to reduce the generalized eigenvalue problem for $\left(A_{1}, B\right)$ to a classical eigenvalue problem for a matrix $A$ which is still a CMV-like matrix modified by a rank-one correction in its first row.

(4) Perform the Fast_QR procedure applied to the input matrix $A$ for the computation of the desired approximations of the zeros of $f(z)$.

The complexity of the algorithm is $O\left(n^{2}\right)$ flops using $O(n)$ memory storage. A Matlab implementation of our composite algorithm has been realized for testing and experimental purposes. The following examples indicate the efficiency and the robustness of the algorithm. In each experiment we compute the finite spectrum of the arrowhead pencil using the Matlab function eig, we match these entries with the approximations returned as output by the Fast_QR procedure and compute the maximum absolute error.

EXAmPLE 4.1. The first numerical example is preparatory. Let us consider the nonlinear equation

$$
f(z)=z^{3} \sin \left(5(z / 2)^{3}\right)=0 .
$$

In table 1 we show the approximations of the multiple zero at the origin obtained by computing the eigenvalues of the arrowhead pencil defined in (2.1), 2.2 with $n=64$ and by the command roots applied to the coefficients of the interpolating polynomial at the same roots of unity. The results confirm some experimental observations in 11 about the better accuracy of the arrowhead linearization compared with the classical companion form for zeros clustered around the origin in the complex plane.

The second preliminary test concerns the robustness of our approach and specifically the accuracy of the $\mathbf{C M V}$ _Reduce procedure. In particular, recall that Remark 2.5 indicates that numerical difficulties can arise for large $n$ when the separation of the nodes approaches zero. We consider the polynomial function

$$
f_{1}(z)=\sum_{j=0}^{n-1}(j+1) z^{j} .
$$

From the Eneström-Kakeya theorem $\left[\mathbf{2 4}\right.$ it follows that the roots of $f_{1}(z)$ live in the interior of the unit disc. In table 2 we show the errors between the finite spectrum of $(A, B)$ and $\left(A_{1}, B\right)$, where $A_{1}$ is generated by means of $\mathbf{C M V} \_$Reduce applied 


\begin{tabular}{c|c} 
pencil_eig & poly_roots \\
\hline $4.7 \mathrm{e}-08+3.4 \mathrm{e}-08 \mathrm{i}$ & $-1.2 \mathrm{e}-03-1.8 \mathrm{e}-03 \mathrm{i}$ \\
\hline $4.7 \mathrm{e}-08+3.4 \mathrm{e}-08 \mathrm{i}$ & $1.2 \mathrm{e}-03+1.8 \mathrm{e}-03 \mathrm{i}$ \\
\hline $4.7 \mathrm{e}-08+3.4 \mathrm{e}-08 \mathrm{i}$ & $-2.2 \mathrm{e}-03+1.6 \mathrm{e}-04 \mathrm{i}$ \\
\hline $4.7 \mathrm{e}-08+3.4 \mathrm{e}-08 \mathrm{i}$ & $2.2 \mathrm{e}-03-1.6 \mathrm{e}-04 \mathrm{i}$ \\
\hline$-1.1 \mathrm{e}-08-1.8 \mathrm{e}-07 \mathrm{i}$ & $9.7 \mathrm{e}-04-2.0 \mathrm{e}-03 \mathrm{i}$ \\
\hline$-1.8 \mathrm{e}-07+4.8 \mathrm{e}-08 \mathrm{i}$ & $-9.7 \mathrm{e}-04+2.0 \mathrm{e}-03 \mathrm{i}$
\end{tabular}

TABLE 1. Approximations of the multiple zero at the origin returned by eig applied to the arrowhead pencil and roots applied to the interpolating polynomial.

\begin{tabular}{c||c|c|c|}
$n$ & 256 & 512 & 1024 \\
\hline $\operatorname{err}(\boldsymbol{z})$ & $6.7 \mathrm{e}-14$ & $1.11 \mathrm{e}-11$ & $2.9 \mathrm{e}-06$ \\
\hline $\operatorname{err}(\hat{\boldsymbol{z}})$ & $\mid 1.4 \mathrm{e}-14$ & $7.8 \mathrm{e}-14$ & $9.4 \mathrm{e}-12$
\end{tabular}

TABLE 2. Error in matching the finite spectra of the pencil in arrow form and the modified pencil under different orderings.

\begin{tabular}{c||c|c|c|c}
$n$ & 128 & 256 & 512 & 1024 \\
\hline err $\| 1.2 \mathrm{e}-14$ & $4.9 \mathrm{e}-14$ & $1.3 \mathrm{e}-13$ & $6.7 \mathrm{e}-11$
\end{tabular}

TABLE 3. Error in matching the spectra of the pencil in arrow form and the perturbed CMV-like matrix.

with the same starting vector $\boldsymbol{w}$ respectively to $D=\operatorname{diag}(\boldsymbol{z}), z_{k}=e^{2 \pi(k-1) / n}$, $1 \leq k \leq n$, and to $D=\operatorname{diag}(\hat{\boldsymbol{z}})$, where $\hat{\boldsymbol{z}}$ is the vector produced by the following reordering of the nodes aimed to distance contiguous points:

$$
z_{2 j-1}=e^{2 \pi(j-1) / n}, \quad z_{2 j}=-z_{2 j-1}, \quad 1 \leq j \leq n / 2 .
$$

Finally, the next table 3 shows the overall errors generated for different values of $n$ starting with the permuted vector of nodes. The condition numbers of the eigenvalues of the perturbed CMV-like matrix of order $n=1024$ are of order $1.0 e+2$ and, therefore, the absolute errors are within the theoretical estimates.

EXAMPLE 4.2. In $\mathbf{1 9}$ the problem of computing the zeros of the following function is considered

$$
f_{2}(z)=z^{50}+z^{12}-5 \sin (20 z) \cos (5 z)-1 .
$$

In Figure 2 we plot the the spectra of the pencil in arrow form and the perturbed CMV-like matrix for different values of $n$.

The test is very hard. The infinity norm of the vector $f(\boldsymbol{z})=\left(f\left(z_{j}\right)\right)$ is of order $9.8 e+13$, the maximum condition number of the eigenvalues of $A^{-1} B$ is 


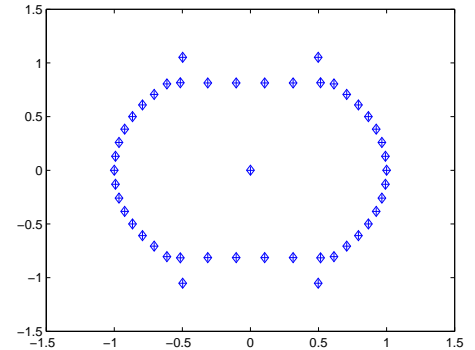

(a) $n=48$

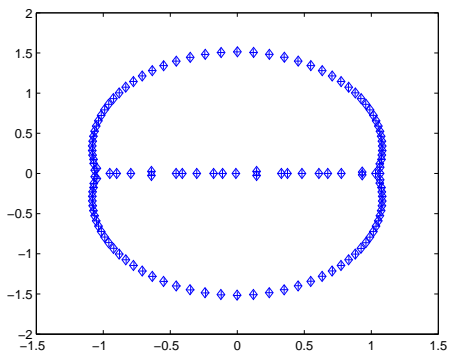

(c) $n=128$

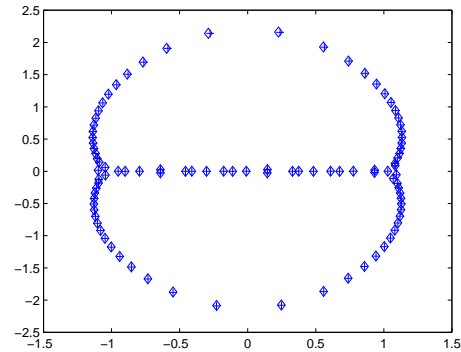

(b) $n=96$

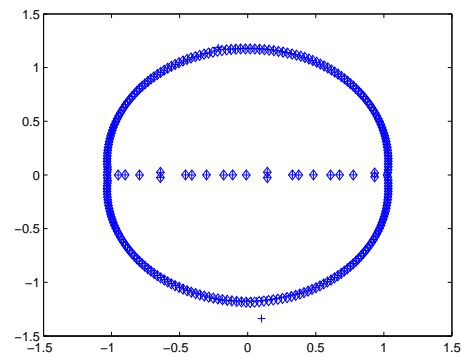

(d) $n=256$

FiguRe 2. Plot of the spectra of the arrowhead pencil (diamond symbol) and the perturbed CMV-like matrix (plus symbol).

of order $1.0 e+8$ and the value of $\sigma$ is of order $1.0 e-08$. It can be observed that for $n=256$ there is a large absolute error in one computed eigenvalue. The condition numbers of the eigenvalues of the perturbed CMV-like matrix range from $1.0 e+8$ to $1.0 e+15$ due to the quite small (but nonzero) value of the $\sigma$ parameter occurring in the deflation procedure. Notwithstanding that, the relevant part of the spectrum, that is, the approximations approaching the zeros of $f_{2}(z)$ are computed quite accurately. By evaluating the residuals we find that there are 21 "good" approximations. In the next figure 3 we illustrate the semi-log scale plot of the residual for the approximations generated by the arrowhead pencil (continuous line) and the perturbed CMV-like matrix (dotted line). 


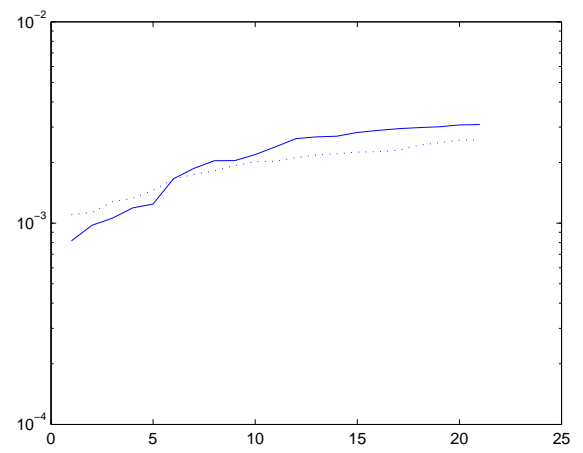

Figure 3. Comparison of the residuals generated by the arrowhead pencil (continuous line) and the perturbed CMV-like matrix (dotted line).

EXAMPLE 4.3. To further investigate the approximation of the spectrum of nonlinear eigenproblems around the origin in the complex plane we consider the scaled Hadeler problem in the NLEVP library [5, that is,

$$
\operatorname{det}(T(z) / \gamma(z))=0, \quad T(z)=\left(e^{z}-1\right) B_{1}+z^{2} B_{2}-B_{0},
$$

where $B_{i} \in \mathbb{R}^{8 \times 8}$ are defined as

$$
B_{0}=100 I_{8},\left(B_{1}\right)_{i, j}=(9-\max (i, j)) i j,\left(B_{2}\right)_{i, j}=1 /(i+j)+8 \delta_{i, j},
$$

with $\delta_{i, j}$ the Kronecker delta symbol and, moreover,

$$
\gamma(z)=\left(\operatorname{det}\left(B_{1}\right)\right)^{1 / 8}\left(e^{z / 2}-1\right)+\left(\operatorname{det}\left(B_{2}\right)\right)^{1 / 8}(z / 2)^{2}+\left(\operatorname{det}\left(B_{0}\right)\right)^{1 / 8} .
$$

It is known that there is a real zero $\lambda \simeq 0.21$ close to the origin $[\mathbf{2 9}$. We compute the eigenvalues of the arrowhead pencil and the perturbed CMV-like matrix for any even $n$ ranging from 64 to 128. From these spectra we determine the best approximations of $\lambda$. The condition numbers of the eigenvalues of the CMV-like matrix range from $1.0 e+07$ to $1.0 e+15$. The parameter $\sigma$ occurring in the deflation procedure is generally small in magnitude (less than $1.0 e-07$ ). For instance, for $n=86$ we find that the infinity norm of $f(\boldsymbol{z})$ is of order $1.1 e+4$ and polyeig applied to $(A, B)$ returns an eigenvalue $\lambda=2.095592506490536 e-01+2.317181655677081 e-13 i$ with estimated condition number of order $1.0 e+5$. The deflation procedure finds a value of $\sigma$ of order $1.0 e-12$. Notwithstanding that, the fast QR algorithm applied to the deflated matrix computes $\lambda=2.095592506545526 e-01+8.022746309423756 e-13 i$. 


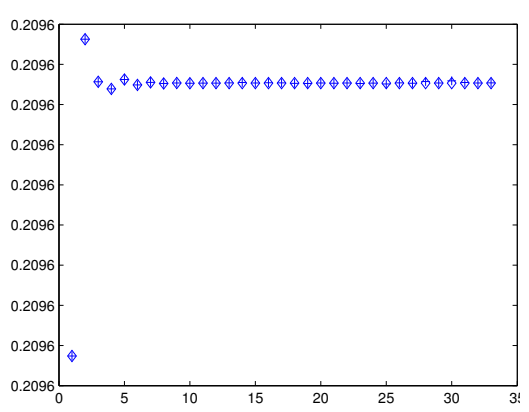

(a) Real part

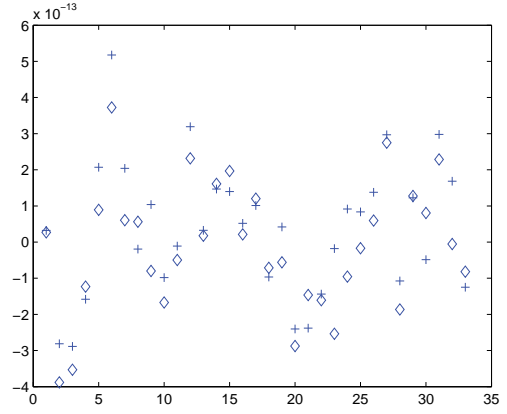

(b) Imaginary part

Figure 4. Approximations of $\lambda$ generated from the spectra of the arrowhead pencil (diamond symbol) and the perturbed CMV-like matrix (plus symbol).

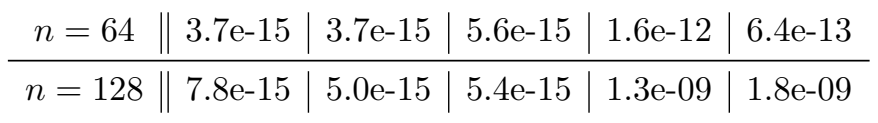

TABLE 4. Errors for the approximations of the five zeros generated starting from the initial pencil in arrow form and the perturbed CMV-like matrix

In the next figure 4 we plot the real and the imaginary parts of the approximations of $\lambda$ computed from the spectra for different values of $n=64+2(j-1)$, $1 \leq j \leq 33$.

We see that the computed approximations of $\lambda$ persist to be accurate independently of the conditioning upper bounds.

EXAMPLE 4.4. The location of the zeros of the holomorphic function

$$
f_{3}(z)=a+b z+z^{2}-h z^{2} e^{-\tau z},
$$

determines the stability of a steady state solution of a neutral functional differential equation 21. Similar models are also used to study the stability of a flow inside an annular combustion chamber [19. The case where $a=1, b=0.5, h=$ $-0.82465048736655, \tau=6.74469732735569$ is analyzed in [26 corresponding to a Hopf bifurcation point. In figure 5 we compare the approximations of the zeros of $f_{3}(z)$ generated from the spectra of the arrowhead pencil and the perturbed CMV-like matrix.

For $n=128$ we find that the infinity norm of $f(\boldsymbol{z})$ is $7.0 e+02$, the magnitude of the parameter $\sigma$ arising in the deflation procedure of order 1.0e-13 and among the considered approximations three eigenvalues of $(A, B)$ have condition numbers of order $1.0 e+03$ while the remaining two eigenvalues have condition numbers of order $1.0 e+08$. In table 4 for $n=64,128$ we report the absolute errors of the five approximations displayed in the figure generated starting from the initial pencil in arrow form and the perturbed CMV-like matrix. 


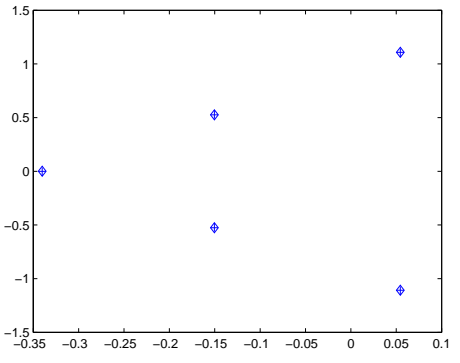

(a) $n=64$

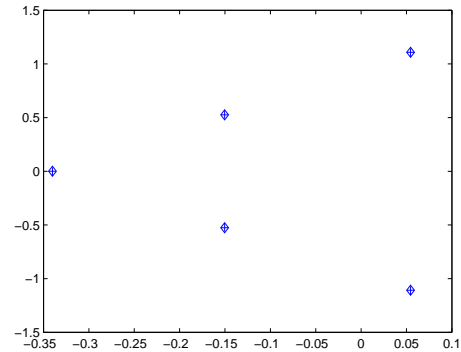

(b) $n=128$

Figure 5. Approximations of the zeros of $f_{3}(z)$ generated from the spectra of the arrowhead pencil (diamond symbol) and the perturbed CMV-like matrix (plus symbol).

EXAmple 4.5. In [4 a mathematical model of cancer growth is proposed which consists of three linear delay differential equations. The stability of the model reduces to investigate the root distribution around the origin in the complex plane of the function

$$
f_{4}(z)=\operatorname{det}\left(z I_{3}-A_{0}-A_{1} e^{-r z}\right)
$$

with

$$
\begin{gathered}
A_{0}=\left[\begin{array}{ccc}
-\mu_{1} & 0 & 0 \\
2 b_{1} & -\left(\mu_{0}+\mu_{Q}\right) & b_{Q} \\
0 & \mu_{Q} & -\left(b_{Q}+\mu_{G_{0}}\right)
\end{array}\right], \\
A_{1}=e^{-\left(\mu_{0}+\mu_{Q}\right) r}\left[\begin{array}{ccc}
2 b_{1} & 0 & b_{Q} \\
-2 b_{1} & 0 & -b_{Q} \\
0 & 0 & 0
\end{array}\right],
\end{gathered}
$$

and

$$
r=5, b_{1}=0.25, b_{Q}=0.2, \mu_{1}=0.28, \mu_{0}=0.11, \mu_{Q}=0.02, \mu_{G_{0}}=0.0001 .
$$

In figure 6 we plot the approximations of the zeros computed by solving the matrix eigenvalue problems.

The approximations returned for the rightmost zero are $\lambda_{\diamond}=6.73 e-04+$ i $9.0 e-15$ and $\lambda_{+}=6.73 e-04+i 2.6 e-13$ which means that the system is unstable. A different selection of the system parameters where we decrease the value of $b_{1}$ corresponding to the duplication rate of tumor cells from $b_{1}=0.25$ to $b_{1}=0.13$ modifies the stability of the system. In the next figure 7 we show the approximated zeros for the novel set of parameters. 


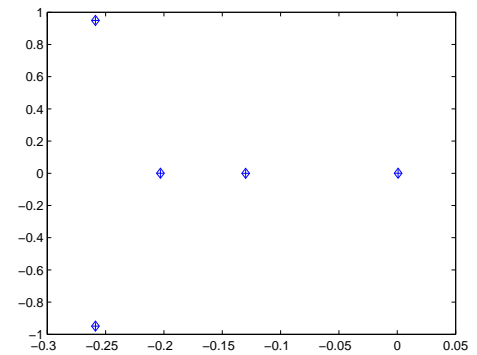

(a) $n=64$

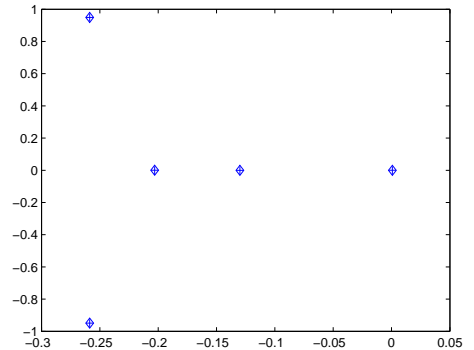

(b) $n=128$

Figure 6. Approximations of the zeros of $f_{4}(z)$ with $b_{1}=0.25$ generated from the spectra of the arrowhead pencil (diamond symbol) and the perturbed CMV-like matrix (plus symbol).

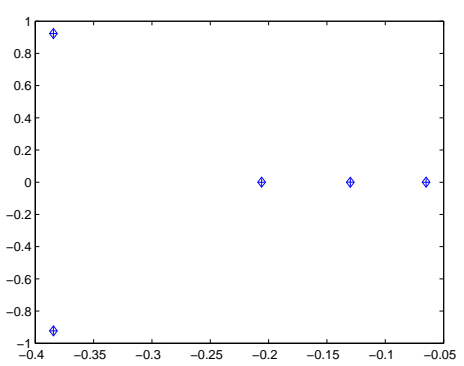

(a) $n=64$

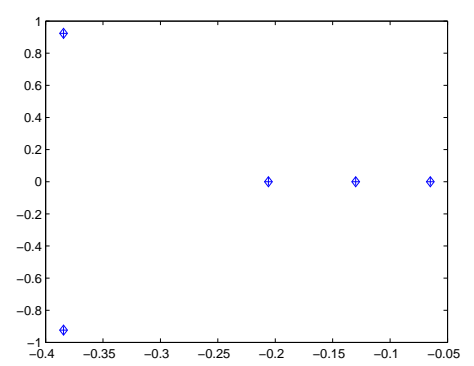

(b) $n=128$

Figure 7. Approximations of the zeros of $f_{4}(z)$ with $b_{1}=0.13$ generated from the spectra of the arrowhead pencil (diamond symbol) and the perturbed CMV-like matrix (plus symbol). 


$$
\begin{array}{c||c|c|c|c|c}
n=64 & 6.5 \mathrm{e}-14 & 1.4 \mathrm{e}-13 & 8.0 \mathrm{e}-14 & 2.0 \mathrm{e}-15 & 1.6 \mathrm{e}-15 \\
\hline n=128 & \mid 1.6 \mathrm{e}-13 & 3.0 \mathrm{e}-13 & 1.5 \mathrm{e}-13 & 2.0 \mathrm{e}-14 & 1.0 \mathrm{e}-14
\end{array}
$$

TABLE 5. Errors for the approximations of the five zeros generated starting from the initial pencil in arrow form and the perturbed CMV-like matrix

The rightmost approximation is $\lambda_{\diamond}=-6.49 e-02+\mathrm{i} 2.3 e-14$ and $\lambda_{+}=$ $-6.49 e-02-i 1.1 e-09$ and the system is stable. The conditioning of the five eigenvalues range from $1.4 e+01$ and $3.1 e+04$. In the next table 5 for $n=64,128$ we show the absolute errors of the five approximations displayed in the figure generated starting from the initial pencil in arrow form and the perturbed CMV-like matrix.

\section{Conclusion and Future Work}

In this paper we have presented a matrix algorithm for the computation of the zeros of an analytic function inside the unit circle in the complex plane. At the heart of the proposed approach there is a fast reduction of the initial generalized eigenproblem in arrow form into a perturbed CMV-like form combined with a fast adaptation of the QR eigenvalue algorithm which exploits the structural properties of this latter formulation. The overall complexity is $O\left(n^{2}\right)$ using $O(n)$ memory storage, where $n$ is the number of interpolation nodes on the unit circle used to discretize the original zerofinding computation. The numerical experience is promising and confirms that the proposed approach performs rather accurately in general. In principle numerical difficulties can be encountered if the nodes are quite close or the deflation procedure provides a small but nonzero cutting parameter. In the first case we think that the problem can be alleviated by a careful analysis of the unitary transformation matrices used in the reduction procedure in order to guarantee the invariance of the rank properties of the transformed matrix. Future work is also concerned with the design of more robust deflation procedures taking into account for the occurrence of tiny but nonzero parameters.

\section{References}

[1] G.S. Ammar, W.B. Gragg, and L. Reichel, On the eigenproblem for orthogonal matrices, Decision and Control, 1986 25th IEEE Conference on, Dec 1986, pp. 1963-1966.

[2] P. Arbenz and G. H. Golub, Matrix shapes invariant under the symmetric QR algorithm, Numer. Linear Algebra Appl. 2 (1995), no. 2, 87-93.

[3] A. P. Austin, P. Kravanja, and L. N. Trefethen, Numerical algorithms based on analytic function values at roots of unity, SIAM J. Numer. Anal. 52 (2014), no. 4, 1795-1821.

[4] M. V. Barbarossa, C. Kuttler, and J. Zinsl, Delay equations modeling the effects of phasespecific drugs and immunotherapy on proliferating tumor cells, Math. Biosci. Eng. 9 (2012), no. $2,241-257$.

[5] T. Betcke, N. J. Higham, V. Mehrmann, C. Schröder, and F. Tisseur, NLEVP: a collection of nonlinear eigenvalue problems, ACM Trans. Math. Software 39 (2013), no. 2, Art. 7, 28.

[6] R. Bevilacqua, G. M. Del Corso, and L. Gemignani, Compression of unitary rank-structured matrices to CMV-like shape with an application to polynomial rootfinding, ArXiv e-prints (2013).

[7] _ A CMV-based eigensolver for companion matrices, ArXiv e-prints (2014), Submitted to SIAM J.Matrix Anal. Appl. 
[8] D. A. Bini, P. Boito, Y. Eidelman, L. Gemignani, and I. Gohberg, A fast implicit QR eigenvalue algorithm for companion matrices, Linear Algebra Appl. 432 (2010), no. 8, 2006-2031.

[9] D. A. Bini, Y. Eidelman, L. Gemignani, and I. Gohberg, Fast QR eigenvalue algorithms for Hessenberg matrices which are rank-one perturbations of unitary matrices, SIAM J. Matrix Anal. Appl. 29 (2007), no. 2, 566-585.

[10] - The unitary completion and $Q R$ iterations for a class of structured matrices, Math. Comp. 77 (2008), no. 261, 353-378.

[11] D. A. Bini and L. Robol, Solving secular and polynomial equations: a multiprecision algorithm, J. Comput. Appl. Math. 272 (2014), 276-292.

[12] P. Boito, Y. Eidelman, and L. Gemignani, Implicit $Q R$ for companion-like pencils, Tech. report, arXiv:1401.5606, 2014. To appear in Math. Comp..

[13] A. Bultheel and M. Van Barel, Vector orthogonal polynomials and least squares approximation, SIAM J. Matrix Anal. Appl. 16 (1995), no. 3, 863-885.

[14] A. Bunse-Gerstner and L. Elsner, Schur parameter pencils for the solution of the unitary eigenproblem, Linear Algebra Appl. 154/156 (1991), 741-778.

[15] M. J. Cantero, L. Moral, and L. Velázquez, Five-diagonal matrices and zeros of orthogonal polynomials on the unit circle, Linear Algebra Appl. 362 (2003), 29-56.

[16] _ Minimal representations of unitary operators and orthogonal polynomials on the unit circle, Linear Algebra Appl. 408 (2005), 40-65.

[17] S. Chandrasekaran, M. Gu, J. Xia, and J. Zhu, A fast QR algorithm for companion matrices, Recent advances in matrix and operator theory, Oper. Theory Adv. Appl., vol. 179, Birkhäuser, Basel, 2008, pp. 111-143.

[18] R. Corless, Generalized companion matrices for the Lagrange basis, Proceedings EACA, 2004.

[19] M. Dellnitz, O. Schütze, and Q. Zheng, Locating all the zeros of an analytic function in one complex variable, J. Comput. Appl. Math. 138 (2002), no. 2, 325-333.

[20] Y. Eidelman, L. Gemignani, and I. Gohberg, Efficient eigenvalue computation for quasiseparable Hermitian matrices under low rank perturbations, Numer. Algorithms 47 (2008), no. 3 , 253-273.

[21] K. Engelborghs, D. Roose, and T. Luzyanina, Bifurcation analysis of periodic solutions of neutral functional-differential equations: a case study, Internat. J. Bifur. Chaos Appl. Sci. Engrg. 8 (1998), no. 10, 1889-1905.

[22] M. Fiedler and T. L. Markham, Completing a matrix when certain entries of its inverse are specified, Linear Algebra Appl. 74 (1986), 225-237.

[23] G. H. Golub and C. F. Van Loan, Matrix computations, third ed., Johns Hopkins Studies in the Mathematical Sciences, Johns Hopkins University Press, Baltimore, MD, 1996.

[24] P. Henrici, Applied and computational complex analysis. Vol. 1, Wiley Classics Library, John Wiley \& Sons, Inc., New York, 1988, Power series—integration-conformal mappinglocation of zeros, Reprint of the 1974 original, A Wiley-Interscience Publication.

[25] R. Killip and I. Nenciu, CMV: the unitary analogue of Jacobi matrices, Comm. Pure Appl. Math. 60 (2007), no. 8, 1148-1188.

[26] P. Kravanja and M. Van Barel, Computing the zeros of analytic functions, Lecture Notes in Mathematics, vol. 1727, Springer-Verlag, Berlin, 2000.

[27] P. W. Lawrence, Fast reduction of generalized companion matrix pairs for barycentric Lagrange interpolants, SIAM J. Matrix Anal. Appl. 34 (2013), no. 3, 1277-1300.

[28] P. W. Lawrence and R. M. Corless, Stability of rootfinding for barycentric Lagrange interpolants, Numer. Algorithms 65 (2014), no. 3, 447-464.

[29] A. Ruhe, Algorithms for the nonlinear eigenvalue problem, SIAM J. Numer. Anal. 10 (1973), 674-689.

[30] Barry S., CMV matrices: five years after, J. Comput. Appl. Math. 208 (2007), no. 1, 120154.

[31] M. Van Barel and A. Bultheel, Orthonormal polynomial vectors and least squares approximation for a discrete inner product, Electron. Trans. Numer. Anal. 3 (1995), no. Mar., 1-23 (electronic).

[32] M. Van Barel, R. Vandebril, P. Van Dooren, and K. Frederix, Implicit double shift QRalgorithm for companion matrices, Numer. Math. 116 (2010), no. 2, 177-212.

[33] R. Vandebril and G.M. Del Corso, An implicit multishift QR-algorithm for Hermitian plus low rank matrices, SIAM Journal on Scientific Computing 32 (2010), no. 4, 2190-2212. 
ZEROFINDING OF ANALYTIC FUNCTIONS BY STRUCTURED MATRIX METHODS 21

Dipartimento di Informatica, Università di Pisa, Largo B. Pontecorvo 3, 56127, Pisa, Italy

E-mail address: 1.gemignani@di.unipi.it 